\title{
THE ESSENTIAL NORMS OF COMPOSITION OPERATORS
}

\section{by BOO RIM CHOE}

(Received 3 October, 1990)

1. Introduction. Throughout the paper $n$ denotes a fixed positive integer unless otherwise specified. Let $B=B_{n}$ denote the open unit ball of $\mathbb{C}^{n}$ and let $S=S_{n}$ denote its boundary, the unit sphere. The unique rotation-invariant probability measure on $S$ will be denoted by $\sigma=\sigma_{n}$. For $n=1$, we use more customary notations $D=B_{1}, T=S_{1}$ and $d \sigma_{1}=d \theta / 2 \pi$. The Hardy space on $B$, denoted by $H^{2}(B)$, is then the space of functions $f$ holomorphic on $B$ for which

$$
\|f\|^{2}=\sup _{0<r<1} \int_{S}|f(r \zeta)|^{2} d \sigma(\zeta)<\infty .
$$

Let $\varphi$ be a holomorphic function on $B$ such that $\varphi(B) \subset D$. For a function $f$ holomorphic on $D$ it is clear that the composite function $f \circ \varphi$ is holomorphic on $B$. If, in addition, $f \in H^{2}(D)$, then $f \circ \varphi \in H^{2}(B)$. This is a consequence of the harmonic majorant characterization of the Hardy spaces (see [13, section 5.6]): a function $g$ holomorphic on $B$ belongs to $H^{2}(B)$ if and only if $|g|^{2}$ has a harmonic majorant. The composition operator $C_{\varphi}: H^{2}(D) \rightarrow H^{2}(B), C_{\varphi} f=f \circ \varphi$, induced by $\varphi$ is therefore bounded by the closed graph theorem.

In case $B=D$ (i.e. $n=1$ ) relations between the operator theoretic properties of $C_{\varphi}$ and the function theoretic properties of the inducing function $\varphi$ have been extensively studied by many authors. See, for example, [5], [16], and references therein. In particular, Shapiro [16] has recently completely answered the question "when is $C_{\varphi}$ compact?" In fact he obtained a complete function theoretic description of the essential norm (=distance to the compact operators) of $C_{\varphi}$ in terms of the Nevanlinna counting function of $\varphi$.

In the present paper we investigate the same question for general $C_{\varphi}$ in terms of the pullback measures $\tau_{\varphi}$ on $\bar{D}$ defined by $\tau_{\varphi}(E)=\sigma\left[\left(\varphi^{*}\right)^{-1}(E)\right]$ (the superscript ${ }^{*}$ denotes the radial limit) for Borel subsets $E$ of $\bar{D}$. By a standard approximation one can easily find

$$
\int_{S} f \circ \varphi^{*} d \sigma=\int_{\bar{D}} f d \tau_{\varphi}
$$

for every Borel function $f \geq 0$ on $\bar{D}$. Note that $(f \circ \varphi)^{*}=f \circ \varphi^{*}[\sigma]$ a.e. on $S$ for functions $f$ continuous on $\bar{D}$. Thus it is easy to see $\left\|C_{\varphi}\right\|=\left\|I_{\tau_{\varphi}}\right\|$ where $I_{\tau_{\varphi}}$ denotes the densely defined inclusion operator of $H^{2}(D)$ into $L^{2}\left(\tau_{\varphi}\right)$. In fact the following is true:

$$
\left\|C_{\varphi}\right\|^{2}=\left\|I_{\tau_{\varphi}}\right\|^{2} \cong\left\|\tau_{\varphi}\right\|_{C}
$$

where $\left\|\tau_{\varphi}\right\|_{C}$ denotes the Carleson norm of $\tau_{\varphi}$ (see Section 2). The notation $\cong$ means that the ratios of two terms are bounded below and above by positive constants $C_{n}$. Here, and elsewhere, $C_{n}$ denotes an absolute constant, depending on the dimension $n$, which may change with each occurrence. The second half of (2) is well known for measures $\tau_{\varphi}$ concentrated on $D$, as the Carleson measure theorem, and remains true for measures $\tau_{\varphi}$

1980 Mathematics Subject Classification (1985 Revision). Primary 47B38, 32A35. Secondary 30 D55.

This research was supported by KOSEF. 
not necessarily concentrated on $D$ (see 3.3). In Section 3 we obtain a complete analogue of (2) for essential norms. In Section 4 we apply this result to calculate the precise essential norms of certain composition operators. Two cases are considered here: the first one involves inner functions and the second one deals with the so-called orthogonal functions. Finally in Section 5 we indicate without detail the analogues in higher dimensional cases and in the Bergman context.

2. Preliminary. For a function $f: B \rightarrow \mathbb{C}$, we let $f^{*}(\zeta)=\lim _{r>1} f(r \zeta)$ if the limit exists at $\zeta \in S$.

It is a well-known fact that if $f \in H^{2}(B)$, then $f^{*}$ exists [ $\left.\sigma\right]$ a.e. on $S$ and the map $f \rightarrow f^{*}$ is a linear isometry of $H^{2}(B)$ into $L^{2}(\sigma)$. We will often use the same letter for a function $f \in H^{2}(B)$ and its boundary function $f \in L^{2}(\sigma)$.

For $\alpha \in B$ we shall let $P_{\alpha}$ denote the test function defined by

$$
P_{\alpha}(z)=\left(\frac{\sqrt{1-|\alpha|^{2}}}{1-\langle z, \alpha\rangle}\right)^{n} \quad(z \in B) .
$$

Here $\langle$,$\rangle denotes the complex inner product on \mathbb{C}^{n}$. By the Cauchy formula on $B$ we have $P_{\alpha} \in H^{2}(B)$ and $\left\|P_{\alpha}\right\|=1$. The Poisson-Szegö kernel on $B$ is the kernel

$$
P(z, \zeta)=\frac{\left(1-|z|^{2}\right)^{n}}{|1-\langle z, \zeta\rangle|^{2 n}} \quad(z \in B, \zeta \in S) .
$$

Note that $P(z, \zeta)=\left|P_{z}(\zeta)\right|^{2}$ for every test function $P_{z}$. The Poisson-Szegö integral $P[\lambda]$ of a complex Borel measure $\lambda$ on $S$ is defined by

$$
P[\lambda](z)=\int_{S} P(z, \zeta) d \lambda(\zeta) \quad(z \in B) .
$$

If $d \lambda=f d \sigma$, then we write $P[\lambda]=P[f]$. The symmetric derivative $\mathscr{D} \lambda$ of a complex Borel measure $\lambda$ on $S$ is defined for $[\sigma]$ a.e. $\zeta \in S$ by

where

$$
\mathscr{D} \lambda(\xi)=\lim _{t \searrow 0} \frac{\lambda(Q(\zeta, t))}{\sigma(Q(\zeta, t))}
$$

$$
Q(\zeta, t)=\{\eta \in S:|1-\langle\eta, \zeta\rangle|<t\}
$$

By Korányi's theorem we have

$$
P[\lambda]^{*}=\mathscr{D} \lambda \quad[\sigma] \text { a.e.. }
$$

This happens at every Lebesgue point of $f$ if $d \lambda=f d \sigma$. Thus $d \lambda=\mathscr{D} \lambda d \sigma$ for $\lambda \ll \sigma$. See [13, Section 5.4] for details.

In what follows the term "measure" will always refer to positive finite Borel measures. Let $\tau$ be a measure on $\bar{B}$. We shall let $I_{\tau}$ denote the densely defined "inclusion" operator of the Hardy space $H^{2}(B)$ into $L^{2}(\tau)$. We also define its "Carleson norm" (see 3.3 below)

$$
\|\tau\|_{C}=\sup \frac{\tau(\Omega(\zeta, t))}{\sigma(Q(\zeta, t))} \quad(\zeta \in S, t>0)
$$


and its "essential Carleson norm"

where

$$
\|\tau\|_{C}=\limsup _{t>0} \sup _{\zeta \in S} \frac{\tau(\Omega(\zeta, t))}{\sigma(Q(\zeta, t))}
$$

$$
\Omega(\zeta, t)=\{z \in \bar{B}:|1-\langle z, \zeta\rangle|<t\} .
$$

A measure on a Borel subset $E$ of $\bar{B}$ will be considered as a measure on $\bar{B}$ concentrated on $E$. For example, $\lambda(\Omega(\zeta, t))=\lambda(Q(\zeta, t))$ for a measure $\lambda$ on $S$.

Given a densely defined linear operator $L$ of a Hilbert space $X$ into another Hilbert space $Y$, we will denote the operator norm of $L$ by $\|L\|$ when there is no possibility of confusion with the $H^{2}$ norm. If $\|L\|<\infty$, we will use the same letter $L$ for the unique bounded extension on all of $X$. The essential norm $\|L\|$ of bounded $L$ is its distance to the compact operators. More precisely,

$$
\|L\|=\inf \{\|L-K\|: K \text { a compact operator } X \text { into } Y\} .
$$

We will not complicate the notation by indicating the spaces explicity in $\|L\|$ and $\|L\|$. This should cause no confusion. Finally, dimensions involved in various notations defined above will be clear from the context.

3. Essential norms. Let $\varphi: B \rightarrow D$ be a holomorphic function and let $\tau_{\varphi}$ be its pullback measure on $\bar{D}$. Since $C_{\varphi}$ is bounded, 1.(1) and a consideration of test functions therefore easily yield $\left\|\tau_{\varphi}\right\|_{C}<\infty$. The same is true for the restriction of $\tau_{\varphi}$ to $T$. With this in mind we prove the following:

Proposition 3.1. Let $\lambda$ be a measure on $S$. Then the following conditions are equivalent.

(i) $\|\lambda\|_{C}<\infty$;

(ii) $\lambda \ll \sigma$ and $\mathscr{D} \lambda \in L^{\infty}(\sigma)$;

(iii) $I_{\lambda}$ is bounded.

If one of the above holds, then

$$
\left\|I_{\lambda}\right\|^{2}=\left\|I_{\lambda}\right\|^{2}=\|D \lambda\|_{\infty} \cong\|\lambda\|_{C} .
$$

Proof. (i) $\Rightarrow$ (ii): Let $M \lambda$ be the maximal function of the measure $\lambda$ defined by

$$
M \lambda(\zeta)=\sup _{t>0} \frac{\lambda(Q(\zeta, t))}{\sigma(Q(\zeta, t))} \quad(\zeta \in S)
$$

By assumption $M \lambda(\zeta) \leq\|\lambda\|_{C}<\infty$ for every $\zeta \in S$ and therefore, by [13, Theorem 5.2.7], $\lambda \ll \sigma$. Clearly, $\|\mathscr{D} \lambda\|_{\infty} \leq\|\lambda\|_{C}$.

(ii) $\Rightarrow$ (iii); Since $\lambda \ll \sigma$, we have $d \lambda=\mathscr{D} \lambda d \sigma$. Consequently, $\left\|I_{\lambda}\right\|^{2} \leq\|\mathscr{D} \lambda\|_{\infty}<\infty$.

(iii) $\Rightarrow$ (i); Apply $I_{\lambda}$ to test functions to derive $\left\|I_{\lambda}\right\|^{2} \geq C_{n}\|\lambda\|_{C}$.

Hence the conditions (i) (iii) are equivalent and, moreover, if one of those three conditions holds, then the proof above shows that $C_{n}\|\lambda\|_{C} \leq\left\|I_{\lambda}\right\|^{2} \leq\|\mathscr{D} \lambda\|_{\infty} \leq\|\lambda\|_{C}$. Thus, to prove (1), it remains to show that $\left\|I_{\lambda}\right\|^{2} \geq\|\mathscr{D} \lambda\|_{\infty}$.

Since $\lim _{|\alpha| \not 1}|h(\alpha)|^{2}\left(1-|\alpha|^{2}\right)^{n}=0$ for every $h \in H^{2}(B)$ (see [13, Theorem 7.2.5]), the sequence of test functions $P_{\alpha}$ converges to 0 weakly on $H^{2}(B)$ as $|\alpha| \lambda 1$ by the Cauchy formula. Let $K: H^{2}(B) \rightarrow L^{2}(\lambda)$ be a compact operator. Since $K$ transforms weakly 
convergent sequences into norm convergent ones (see [19, Theorem 6.3]), we have

$$
\lim _{|\alpha| \wedge 1}\left\|K P_{\alpha}\right\|_{2}=0
$$

where \|\|$_{2}$ denotes the $L^{2}(\lambda)$ norm. Write $L=I_{\lambda}$ for notational simplicitly. Then, for every $\alpha \in B$,

$$
\|L-K\| \geq\left\|(L-K) P_{\alpha}\right\|_{2} \geq\left\|L P_{\alpha}\right\|_{2}-\left\|K P_{\alpha}\right\|_{2},
$$

so by (1),

$$
\|L-K\| \geq \limsup _{|\alpha| \lambda_{1}}\left\|L P_{\alpha}\right\|_{2} .
$$

Taking the infimum on the left side of the above over all $K$ and writing $\alpha=(1-t) \zeta$ $(\zeta \in S, 0<t<1)$, we obtain

$$
\|L\|^{2} \geq \lim \sup _{\triangle 0} \sup _{\zeta \in S} \int_{S}\left|P_{\alpha}\right|^{2} d \lambda
$$

Thus we have

$$
\|L\|^{2} \geq \limsup _{t>0} P[\lambda]((1-t) \zeta) \quad(\zeta \in S) .
$$

This completes the proof by 2.(1).

The following lemma is originally due to Ryff $[15]$ in the setting $B=D$. A similar lemma appears in [8].

Lemma 3.2. Let $\varphi: B \rightarrow D$ be a holomorphic function and assume $f \in H^{2}(D)$. Then

$$
(f \circ \varphi)^{*}=\tilde{f} \circ \varphi^{*} \quad[\sigma] \text { a.e. }
$$

where $\tilde{f}=f$ on $D$ and $\tilde{f}=f^{*}$ on $T$.

Proof. First of all, note that the lemma is true if $\bar{f}$ is continuous on $\bar{D}$. Let $f_{r}$ $(0<r<1)$ be the dilated function $z \rightarrow f(r z)$ on $D$. Then $f_{r} \rightarrow f$ in $H^{2}(D)$ and thus

$$
\lim _{r>1} \int_{S}\left|(f \circ \varphi)^{*}-\tilde{f}_{r} \circ \varphi^{*}\right|^{2} d \sigma=0 .
$$

Let $\lambda$ be the restriction of $\tau_{\varphi}$ to $T$. Since $\|\lambda\|_{C}<\infty$ as remarked earlier, $f^{*}$ exists [ $[\lambda]$ a.e. on $T$ by Proposition 3.1. From this fact one easily obtains

$$
\lim _{r \rightarrow 1} \tilde{f}_{r} \circ \varphi^{*}(\zeta)=\tilde{f} \circ \varphi^{*}(\zeta) \text { for }[\sigma] \text { a.e. } \zeta \in S \text {. }
$$

An application of Fatou's lemma now shows the lemma by (1) and (2).

3.3. Carleson Measures. A measure $\tau$ on $B$ such that $\|\tau\|_{C}<\infty$ is called a Carleson measure. The importance of Carleson measures lies in the so-called Carleson measure theorem ([1], [8]; also [4], [11]):

$$
\left\|I_{\tau}\right\|^{2} \cong\|\tau\|_{C}
$$


holds for measures $\tau$ on $B$. What is needed here is a slight variation of this result, where $\tau$ is not necessarily concentrated on $B$. In this setting the inequality $\left\|I_{\tau}\right\|^{2} \geq C_{n}\|\tau\|_{C}$ follows as before by considering test functions. For the other direction of the inequality, suppose $\|\tau\|_{C}<\infty$. Let $\mu$ and $\lambda$ be the restrictions of $\tau$ to $B$ and $S$, respectively. Then it is easily verified from (1) and Proposition 3.1 that

$$
\left\|I_{\tau}\right\|^{2} \leq\left\|I_{\mu}\right\|^{2}+\left\|I_{\lambda}\right\|^{2} \cong\|\mu\|_{C}+\|\lambda\|_{C} \leq 2\|\tau\|_{C} .
$$

The following must have been also well known to some workers in the field. We include it for the sake of completeness.

Proposition 3.4. $\left\|I_{\tau}\right\|^{2} \cong\|\tau\|_{C}$ holds for measures $\tau$ on $\bar{B}$.

Proof. Write $L=I_{\tau}$ again for notational simplicity. Then, as in the proof of Proposition 3.1, we have

$$
\|L\|^{2} \geq \lim \sup _{\supset 0} \sup _{\zeta \in S} \int_{\bar{B}}\left|P_{(1-t) \zeta}\right|^{2} d \tau .
$$

Replace $\bar{B}$ by $\Omega(\zeta, t)$ in the integral of the above. Then the resulting integral is easily seen to be bounded below by $C_{n} \tau(\Omega(\zeta, t)) t^{-n}$. Since (see [13, Proposition 5.1.4])

$$
\sigma(Q(\zeta, t)) \cong t^{n} \quad(0<t<2)
$$

we obtain

$$
\|L\|^{2} \geq C_{n}\|\tau\|_{c}
$$

Now we prove the other direction of the inequality. For $0<r<1$, let $L_{\tau}=L_{\tau}$, where $\tau_{r}$ denotes the restriction of $\tau$ to the annulus $\bar{B} \backslash(1-r) \bar{B}$ and let $M_{r}: L^{2}(\tau) \rightarrow L^{2}(\tau)$ be the multiplication by the characteristic function of $(1-r) \bar{B}$. Then $M_{r} \circ L$ is compact (by a normal family argument). Thus

$$
\|L\| \leq\left\|L-M_{r} \circ L\right\|=\left\|L_{r}\right\|
$$

and therefore, since the sequence $\left\|L_{r}\right\|$ is monotone, we have

$$
\|L\| \leq \lim _{r>0}\left\|L_{r}\right\| .
$$

Hence the following inequality will finish the proof by (2) and 3.3.(1):

$$
\left\|\tau_{r}\right\|_{C} \leq C_{n}\|\tau\|_{C}+o(1) \quad(r \searrow 0) .
$$

The above is implicit in the proof of Theorem 1.1 of [8] in case $\|\tau\|_{C}=0$ and a slight modification shows the general case. We include it for completeness. Fix $\zeta \in S$. We need verify

$$
\left.\sup _{0<t<2} \frac{\tau_{r}(\Omega(\zeta, t))}{\sigma(Q(\zeta, t))} \leq C_{n}\|\tau\|_{C}+o(1) \quad \text { (uniformly in } \zeta\right) .
$$

Since $\tau_{r}(\Omega(\zeta, t))=\tau(\Omega(\zeta, t))$ for $t \leq r$,

$$
\left.\sup _{0<t \leq r} \frac{\tau_{r}(\Omega(\zeta, t))}{\sigma(Q(\zeta, t))} \leq\|\tau\|_{C}+o(1) \quad \text { (uniformly in } \zeta\right) .
$$


Now assume $r<t<2$. Before proceeding, we introduce a temporary notation:

$$
D(\zeta, \delta)=\{z \in \bar{B}:|z|>1-\delta, z /|z| \in Q(\zeta, \delta)\} .
$$

Note that $\Omega(\zeta, \delta) \subset D(\zeta, \delta) \subset \Omega(\zeta, 2 \delta)$. Let $\left\{\eta_{1}, \eta_{2}, \ldots, \eta_{N}\right\}$ be a maximal collection of points in $Q(\zeta, t)$ subject to the condition $\left|1-\left\langle\eta_{i}, \eta_{j}\right\rangle\right| \geqslant r$ for $i \neq j$. By the triangle inequality (see [13, Proposition 5.1.2.])

$$
|1-\langle a, b\rangle|^{1 / 2} \leq|1-\langle a, c\rangle|^{1 / 2}+|1-\langle b, c\rangle|^{1 / 2} \quad(a, b, c \in \bar{B}),
$$

the balls $Q\left(\eta_{j}, r / 4\right)$ are pairwise disjoint and contained in $Q(\zeta, 3 t)$. Hence $N \leq C_{n}(t / r)^{n}$ by (1). On the other hand, the balls $Q\left(\eta_{j}, r\right)$ cover $Q(\zeta, t)$ by maximality and thus

$$
A_{r} \cap \Omega(\zeta, t) \subset A_{r} \cap D(\zeta, t) \subset \bigcup_{j=1}^{N} D\left(\eta_{j}, r\right) \subset \bigcup_{j=1}^{N} \Omega\left(\eta_{j}, 2 r\right) .
$$

Since $N \leq C_{n}(t / r)^{n}$, the above combined with (1) implies

$$
\sup _{r<t<2} \frac{\tau_{r}(\Omega(\zeta, t))}{\sigma(Q(\zeta, t))} \leq C_{n} \sup _{\eta \in S} \frac{\tau(\Omega(\eta, 2 r))}{\sigma(Q(\eta, 2 r))} .
$$

The right side of the above is dominated by $C_{n}\|\tau\|_{C}+o(1)$ where $o(1)$ is independent of $\zeta$. This, together with (4), shows (3).

We are now ready to prove the main result of this section.

THEOREM 3.5. Let $\varphi: B \rightarrow D$ be a holomorphic function. Then

$$
\left\|C_{\varphi} \mid\right\|^{2}=\left\|I_{\tau_{\varphi}}\right\|^{2} \cong\left\|\tau_{\varphi}\right\|_{C} .
$$

Proof. For simplicity write $\tau=\tau_{\varphi}$. Recall that $I_{\tau}$ is bounded. Let $R: H^{2}(B) \rightarrow L^{2}(\sigma)$ be the isometry defined by $R f=f^{*}$ and let $W: L^{2}(\tau) \rightarrow L^{2}(\sigma)$ be the isometry defined by $W g=g \circ \varphi^{*}$. Then Lemma 3.2 is simply

$$
R \circ C_{\varphi}=W \circ I_{\tau} .
$$

Now let $W^{*}: L^{2}(\sigma) \rightarrow L^{2}(\tau)$ be the adjoint of $W$ defined by the requirement

$$
\int_{S} f \cdot \overline{W g} d \sigma=\int_{\bar{D}} W^{*} f \cdot \bar{g} d \tau .
$$

It is easy to find from 1.(1) that $W^{*} \circ W$ is the identity on $L^{2}(\tau)$. Also note that $W^{*}$ is norm-decreasing. Hence, for a compact operator $K: H^{2}(D) \rightarrow H^{2}(B)$, we obtain from (1)

$$
\left\|C_{\varphi}-K\right\|=\left\|W \circ I_{\tau}-R \circ K\right\| \geq\left\|I_{\tau}-W^{*} \circ R \circ K\right\| \geq\left\|I_{\tau}\right\| .
$$

Upon taking infimum over all $K$, we find $\left\|C_{\varphi}\right\| \geq\left\|I_{\tau}\right\|$. By using the Cauchy transform $C: L^{2}(\sigma) \rightarrow H^{2}(B)$ (see $[13$, Section 6.3]) one can prove

$$
\left\|I_{\tau}-K\right\| \geq\left\|C_{\varphi}-C \circ W \circ K\right\| \geq\left\|C_{\varphi}\right\|
$$

in a similar way for any compact operator $K: H^{2}(D) \rightarrow L^{2}(\tau)$ and thus $\left\|I_{\tau}\right\| \geq\left\|C_{\varphi}\right\|$. Consequently, $\left\|C_{\varphi}\right\|^{2}=\left\|I_{\tau}\right\|^{2} \cong\|\tau\|_{C}$ by Proposition 3.4.

COROLlaRY 3.6. For a holomorphic function $\varphi: B \rightarrow D, C_{\varphi}$ is compact if and only if $\left\|\tau_{\varphi}\right\|_{C}=0$. 
4. Two special cases. The explicit value of the norm or essential norm of $C_{\varphi}$ has been known only for very special classes of inducing functions $\varphi$. See [5], [10] and [16]. In this section we calculate the precise essential norms of composition operators induced by two special classes of functions.

The first one involves inner functions. Recall that a holomorphic function $\varphi: B \rightarrow D$ is called inner if $\left|\varphi^{*}\right|=1[\sigma]$ a.e.. The norm of a composition operator $C_{\varphi}$ induced by an inner function $\varphi$ on $D$ was calculated by Nordgren ([10, Theorem 1]):

$$
\left\|C_{\varphi}\right\|^{2}=\frac{1+|\varphi(0)|}{1-|\varphi(0)|}
$$

Recently Shapiro ([16, Theorem 2.5]) has proved that such a composition operator has the same essential norm as above by using his remarkable essential norm formula ([16, Theorem 2.3]) for arbitrary composition operators on $H^{2}(D)$. It is an interesting fact that the (essential) norm formula for $C_{\varphi}$ induced by an inner function $\varphi$ is independent of dimensions. This is contained in Theorem 4.2. Our derivation is independent. We first prove a lemma.

Lemma 4.1. Suppose $\tau$ is a measure on $B$ and let $\tau_{r}(0<r<1)$ be its restriction to the annulus $B \backslash(1-r) \bar{B}$. Then

$$
\left\|I_{\tau}\right\|\left\|=\lim _{r \supset 0}\right\| I_{\tau_{r}} \|
$$

Proof. Put $L=I_{\tau}$ and $L_{r}=I_{\tau}$. Let $K: H^{2}(B) \rightarrow L^{2}(\tau)$ be an arbitrary compact operator and let $N_{r}: L^{2}(\tau) \rightarrow L^{2}(\tau)$ be the multiplication by the characteristic function of $B \backslash(1-r) \bar{B}$. Then, since $\left\|N_{r}\right\| \leq 1$, we have

$$
\|L-K\| \geq\left\|N_{r} \circ L-N_{r} \circ K\right\| \geq\left\|N_{r} \circ L\right\|-\left\|N_{r} \circ K\right\|=\left\|L_{r}\right\|-\left\|N_{r} \circ K\right\| .
$$

The sequence $N_{r}$ is uniformly bounded and converges to 0 pointwise on $L^{2}(\tau)$ as $r \searrow 0$. It is therefore equicontinuous and converges to 0 uniformly on compact subsets of $L^{2}(\tau)$ by Ascoli's theorem. In particular, this happens on $K(\Phi)$ where $\Phi$ is the closed unit ball of $H^{2}(B)$. This means $\lim _{r \succ 0}\left\|N_{r} \circ K\right\|=0$. It follows that $\|L-K\| \geq \lim _{r \searrow 0}\left\|L_{r}\right\|$ and thus $\|L\| \geq \lim _{r \searrow 0}\left\|L_{r}\right\|$. For the other direction, see 3.4(2).

THEOREM 4.2. Suppose $\varphi$ is an inner function on $B$. For $0<t \leq 1$, let $\psi=(1-t) b+$ $t \varphi$ where $b$ is a unimodular number such that $b|\varphi(0)|=\varphi(0)$. Then

$$
\left\|C_{\psi}\right\|^{2}=\left\|C_{\psi}\right\|^{2}=\frac{1}{t} \cdot \frac{1+|\varphi(0)|}{1-|\varphi(0)|} .
$$

Proof. Let $P\left(z, e^{i \theta}\right)$ be the Poisson kernel on $D$. Since $\varphi$ is an inner function, we have $([\mathbf{1 0}],[12])$

$$
d \tau_{\varphi}(\theta)=P\left(a, e^{i \theta}\right) \frac{d \theta}{2 \pi} \quad(a=\varphi(0)) .
$$

The pullback measure $\tau=\tau_{\psi}$ is therefore a Carleson measure on $D$ such that

$$
\int_{D} f d \tau=\int_{0}^{2 \pi} f \circ h\left(e^{i \theta}\right) P\left(a, e^{i \theta}\right) \frac{d \theta}{2 \pi}
$$


for every Borel function $f \geq 0$ on $D$ where $h(z)=(1-t) b+t z$ for $z \in D$. Hence the theorem is a special case of the following result by Theorem 3.5.

We continue using the notation defined in the proof above.

THEOREM 4.3. Put $\tau_{1}=\tau$ and let $\tau_{r}(0<r<1)$ be the restriction of $\tau$ to the annulus $D \backslash(1-r) \bar{D}$. Then

$$
\left\|I_{\tau_{r}}\right\|^{2}=\left\|\mid I_{\tau_{r}}\right\|^{2}=\left\|\tau_{r}\right\|_{C}=\frac{1}{t} \cdot \frac{1+|a|}{1-|a|} \quad(0<r \leq 1) .
$$

Note: This shows that the equality can happen in Proposition 3.4 in a nontrivial way.

Proof. Write $L_{r}=I_{\tau_{r}}$. By Cowen's norm formula ([5, Theorem 3]) we have $\left\|C_{h}\right\|^{2}=t^{-1}$. Hence it is clear from 4.2(1) that

$$
\left\|L_{r}\right\|^{2} \leq\left\|C_{h}\right\|^{2} \cdot \frac{1+|a|}{1-|a|}=\frac{1}{t} \cdot \frac{1+|a|}{1-|a|} .
$$

On the other hand, considering test functions on $D$, we have

$$
\left\|L_{r}\right\|^{2} \geq \int_{0}^{2 \pi}\left|P_{\alpha}\left(h\left(e^{i \theta}\right)\right)\right|^{2} \chi_{r}\left(e^{i \theta}\right) P\left(a, e^{i \theta}\right) \frac{d \theta}{2 \pi} \quad(\alpha \in D)
$$

where $\chi_{r}$ denotes the characteristic function of the set of points $e^{i \theta}$ such that $\left|h\left(e^{i \theta}\right)\right|>r$. For $\alpha=s b(0<s<1)$ a straightforward calculation shows

$$
\left|P_{\alpha}\left(h\left(e^{i \theta}\right)\right)\right|^{2}=\frac{1-s}{1-s+2 t s} P\left(\frac{t s}{1-s+t s} b, e^{i \theta}\right) .
$$

Inserting the above into (2), we find

$$
\left\|L_{r}\right\|^{2} \geq \frac{1+s}{1-s+2 t s} P\left[\chi_{r} . P(a, \cdot)\right]\left(\frac{t s}{1-s+t s} b\right) .
$$

Take the limits $s \rightarrow 1$ on the right ride of the above and use 2.(1) to obtain

$$
\left\|L_{r}\right\|^{2} \geq t^{-1} \chi_{r}(b) P(a, b)=\frac{1}{t} \cdot \frac{1+|a|}{1-|a|} .
$$

Thus, by (1), (3) and Lemma 4.1,

$$
\left\|L_{r}\right\|^{2}=\left\|L_{r}\right\|^{2}=\frac{1}{t} \cdot \frac{1+|a|}{1-|a|} .
$$

It remains to compute $\left\|\tau_{r}\right\|_{c}$. Note that

$$
\sup _{\theta} \tau\left(\Omega\left(e^{i \theta}, s\right)\right)=\tau(\Omega(b, s))
$$

and that

$$
h\left(e^{i \theta}\right) \in \Omega(b, s) \Leftrightarrow e^{i \theta} \in Q_{s / t}
$$


Here $Q_{\delta}=Q(b, \delta)$ for $\delta>0$. Also note that $\lim _{\delta \backslash 0} \delta^{-1}\left|Q_{\delta}\right|=\pi^{-1}$ where $\left|Q_{\delta}\right|$ denotes the normalized arclength of $Q_{\delta}$. It follows from these observations and 4.2.(1) that

$$
\|\tau\|_{C}=\frac{1}{t} \lim _{\delta \searrow 0} \frac{1}{\left|Q_{\delta}\right|} \int_{Q_{\delta}} P\left(a, e^{i \theta}\right) \frac{d \theta}{2 \pi}=\frac{1}{t} \cdot \frac{1+|a|}{1-|a|} .
$$

The second equality of the above holds by continuity of $P(a, \cdot)$ at $b$. This completes the proof because $\|\tau\|_{C}=\left\|\tau_{r}\right\|_{C}$ by definition.

We now turn to the so-called orthogonal functions. We shall use the notation

$$
E_{\varphi}=\left\{\zeta \in S: \varphi^{*}(\zeta) \text { exists and is unimodular }\right\}
$$

4.4. Orthogonal Functions. Let us say that a holomorphic function $\varphi: B \rightarrow D$ is orthogonal if the sequence $1, \varphi, \varphi^{2}, \ldots$ forms an orthogonal set in $H^{2}(B)$. It is clear that the rotation-invariance of $\tau_{\varphi}$ implies the orthogonality of $\varphi$. It is less clear that the converse is also true: the orthogonality of $\varphi$ implies the rotation-invariance of $\tau_{\varphi}$. This follows from the fact that if $\varphi$ is orthogonal, then

$$
\int_{\bar{D}} h\left(e^{i \theta} z\right) d \tau_{\varphi}(z)=\int_{\bar{D}} h(z) d \tau_{\varphi}(z)
$$

holds for $\theta$ real and functions $h$ of the form $h(z)=z^{k} \bar{z}^{l}(k, l \geq 0)$ which span a dense subset of $L^{\prime}\left(\tau_{\varphi}\right)$. It is easy to see that if $\varphi$ is an inner function on $B$ with $\varphi(0)=0$, then $\varphi$ is orthogonal. Also, every nonconstant (holomorphic) homogeneous polynomial $\varphi: B \rightarrow$ $D$ is orthogonal. There are others: for $n \geq 2$ one may construct orthogonal functions by using the integral formula

$$
\int_{S} f d \sigma=\left(\begin{array}{c}
n-1 \\
k
\end{array}\right) \int_{B_{k}}\left(1-|z|^{2}\right)^{l-1} d V_{k}(z) \int_{S_{l}} f\left(z, \sqrt{1-|z|^{2}} \zeta\right) d \sigma_{l}(\zeta)
$$

where $k+l=n, f \in L^{1}(\sigma)$, and $V_{k}$ denotes the normalized volume measure on $B_{k}$. If $k=n-1$, then (1) is Proposition 1.4.7 of [13]. This general form can be shown by the "same" proof. Also, the orthogonality is invariant under compositions with measure preserving maps ([14, Chapter 14], [18]). Moreover, the author has been informed that Jose Fernandez has constructed orthogonal functions $\varphi$, on $D$ and hence on $B$, for which $0<\sigma\left(E_{\varphi}\right)<1$.

LeMma 4.5. If $\tau$ is a rotation-invariant measure on $\bar{B}$, then $\|\tau\|_{C} \leq \tau(S)$.

Proof. Since $\tau$ is rotation-invariant, we have, for $e_{1}=(1,0, \ldots, 0) \in S$,

$$
\tau\left(\Omega\left(e_{1}, t\right)\right)=\tau(\Omega(\zeta, t)) \quad(\zeta \in S, t>0) .
$$

Let $A_{t}=\bar{B} \backslash(1-t) \bar{B}(0<t<1)$. Then $\Omega(\zeta, t) \subset A_{t}$ and thus, by (1) and Fubini's theorem,

$$
\tau\left(\Omega\left(e_{1}, t\right)\right)=\int_{S} \tau(\Omega(\zeta, t)) d \sigma(\zeta)=\int_{A_{t}} \sigma(E(z, t)) d \tau(z)
$$

where $E(z, t)=\{\zeta \in S:|1-\langle z, \zeta\rangle|<t\}$. Note that $E(z, t) \subset Q(z /|z|, t)$ and thus 
$\sigma(E(z, t)) \leq \sigma\left(Q\left(e_{1}, t\right)\right)$ for every $z \in A_{t}$. Thus, by (2),

$$
\tau\left(\Omega\left(e_{1}, t\right)\right) \leq \tau\left(A_{t}\right) \sigma\left(Q\left(e_{1}, t\right)\right) .
$$

This implies $\|\tau\|_{C} \leq \lim _{t \searrow 0} \tau\left(A_{t}\right)=\tau\left(\bigcap A_{t}\right)=\tau(S)$.

Lemma 4.6. Suppose $\tau$ is a measure on $\bar{B}$ such that $\|\tau\|_{C}<\infty$. Let $\mu$ and $\lambda$ be its restrictions to $B$ and $S$, respectively.

(i) If $\|\mu\|_{C}=0$, then $\left\|I_{\tau}\right\|^{2}=\|D \lambda\|_{\infty}$.

(ii) If $\tau$ is rotation-invariant, then $\left\|I_{\tau}\right\|^{2}=\tau(S)$.

Proof. By considering test functions as before, one can easily obtain $\left\|I_{\tau}\right\|^{2} \geq\|\mathscr{D} \lambda\|_{\infty}$ from 2.(1). Suppose $\|\mu\|_{C}=0$ and let $M_{B}: L^{2}(\tau) \rightarrow L^{2}(\tau)$ be the multiplication by the characteristic function of $B$. Then one easily obtains $\left\|M_{B} \circ I_{\tau}\right\|\|=\| I_{\mu} \|$. Thus, by Proposition $3.4, M_{B} \circ I_{\tau}$ is compact and hence $\left\|I_{\tau}\right\| \leq\left\|I_{\tau}-M_{B} \circ I_{\tau}\right\|=\left\|I_{\lambda}\right\|$ so that (i) holds by Proposition 3.1 .

Now assume $\tau$ is rotation-invariant. Then $\mu$ and $\lambda$ are also rotation-invariant. Thus $\|\mu\|_{C}=0$ by Lemma 4.5 and $d \lambda=\lambda(S) d \sigma$ by the uniqueness of the rotation-invariant probability measure on $S$. Accordingly, $\left\|I_{\tau}\right\|^{2}=\|\mathscr{D} \lambda\|_{\infty}=\lambda(S)=\tau(S)$ by (i).

Note that $\tau_{\varphi}(T)=\sigma\left(E_{\varphi}\right)$. Thus, combining Theorem 3.5 and Lemma 4.6, we obtain

THEOREM 4.7. If $\varphi$ is an orthogonal function on $B$, then $\left\|C_{\varphi}\right\|^{2}=\sigma\left(E_{\varphi}\right)$.

For $n \geq 2$ there is a sufficient condition which implies $\sigma\left(E_{\varphi}\right)=0$ for holomorphic functions $\varphi: B \rightarrow D$. For references concerning this topic, see [14, Section 15.3]. For example, if $\varphi$ is continuously differentiable up to the boundary, then $\sigma\left(E_{\varphi}\right)=0$. Such a function therefore induces a compact composition operator whenever it is orthogonal by Theorem 4.7. In particular, every homogeneous polynomial that maps $B$ onto $D$ induces a compact composition operator. This composition operator, however, is not always a Hilbert-Schmidt operator as the following example shows. Note that $C_{\varphi}$ is a HilbertSchmidt operator if and only if $\left(1-|\varphi|^{2}\right)^{-1} \in L^{1}(\sigma)$.

EXAMPLE 4.8. Let $\varphi: B_{2} \rightarrow D$ be an orthogonal function, with $\varphi\left(B_{2}\right)=D$, taking one of the following forms:

(i) $\varphi\left(z_{1}, z_{2}\right)=z_{1}^{\alpha_{1}}\left(1-z_{2}^{\alpha_{2}}\right)^{-1} \quad\left(\alpha_{1}, \alpha_{2} \geq 2\right)$;

(ii) $\varphi\left(z_{1}, z_{2}\right)=a_{1} z_{1}^{d}+a_{2} z_{2}^{d}$

(iii) $\varphi\left(z_{1}, z_{2}\right)=b z_{1}^{\alpha_{1}} z_{2}^{\alpha_{2}}$.

Note that $\sigma\left(E_{\varphi}\right)=0$ and thus $C_{\varphi}$ is compact by Theorem 4.7. For such a function $\varphi$, we have $\tau_{\varphi} \ll m(m$ denotes the area measure on $D)$ and

$$
\frac{d \tau_{\varphi}}{d m}(z)=c\left(1-|z|^{2}\right)^{\left(1-\operatorname{dim} E_{\varphi}\right) / 2}[1+o(1)] \quad(|z| \nearrow 1)
$$

where $c=c(\varphi)>0$ and dim refers to the topological dimension. See [2] and [3]. Since $E_{\varphi}$ contains a circle, $\operatorname{dim} E_{\varphi} \geq 1$ and therefore

$$
\int_{S_{2}} \frac{d \sigma_{2}}{1-|\varphi|^{2}}=\int_{D} \frac{d \tau_{\varphi}(z)}{1-|z|^{2}}=\infty
$$

In other words, $C_{\varphi}$ is not a Hilbert-Schmidt operator. 
5. Remarks. Note that the a priori boundedness of $C_{\varphi}$ plays an essential role in the argument used in Section 3, especially in the proof of Lemma 3.2. The situation is different in higher dimensional cases. The densely defined composition operator $C_{\Phi}$ of $H^{2}\left(B_{k}\right)$ into $H^{2}\left(B_{n}\right)$ induced by a general holomorphic map $\Phi: B_{n} \rightarrow B_{k}$ is not necessarily bounded; see for example [8]. Nevertheless, it is not hard to see that the analogue of 1.(2) still remains true:

$$
\left\|C_{\Phi}\right\|^{2}=\left\|I_{\tau_{\Phi}}\right\|^{2} \cong\left\|\tau_{\Phi}\right\|_{C}
$$

where $\tau_{\Phi}=\sigma_{n}\left[\left(\Phi^{*}\right)^{-1}\right]$ (* denotes the radial limit) is the pullback meaure of $\Phi$ as before. Now, once the boundedness of $C_{\Phi}$ is known, one can easily prove an analogue of Lemma 3.2. The rest of the proof of the following theorem remains the same:

THEOREM 5.1. Suppose a holomorphic map $\Phi: B_{n} \rightarrow B_{k}$ induces a bounded composition operator $C_{\Phi}: H^{2}\left(B_{k}\right) \rightarrow H^{2}\left(B_{n}\right)$. Then $\left\|C_{\Phi}\right\|\left\|^{2}=\right\| I_{\tau_{\Phi}}\|\|^{2} \cong\left\|\tau_{\Phi}\right\| \|_{C}$.

The following consequence of Theorem 5.1 appears in [8] in case $k=n$.

Corollary 5.2. Let $C_{\Phi}$ be the composition operator induced by a holomorphic map $\Phi: B_{n} \rightarrow B_{k}$. Then $C_{\Phi}$ is compact if and only if $\left\|\tau_{\Phi}\right\|_{C}=0$.

Using the above corollary, we give a class of holomorphic maps that induces compact composition operators.

EXAMPLE 5.3. Let $\Phi: B_{n+m} \rightarrow B_{m}$ be a holomorphic map of the form:

$$
\Phi(z, w)=\varphi(z) w \quad\left(|z|^{2}+|w|^{2}<1, z \in \mathbb{C}^{n}, w \in \mathbb{C}^{m}\right)
$$

where $\varphi: B_{n} \rightarrow \mathbb{C}$ is a (possibly unbounded) holomorphic function. Then, by 4.4.(1),

$$
\int_{\bar{B}_{m}} f d \tau_{\Phi}=\int_{B_{n}} d \mu(z) \int_{S_{m}} f\left(\varphi(z) \sqrt{1-|z|^{2}} \zeta\right) d \sigma_{m}(\zeta)
$$

where $f \in L^{1}\left(\tau_{\Phi}\right)$ and $d \mu(z)=\left(\begin{array}{c}n+m-1 \\ n\end{array}\right)\left(1-|z|^{2}\right)^{m-1} d V_{n}(z)$. Let $E_{\Phi}$ be the set of points $\eta \in S_{n+m}$ such that $\left|\Phi^{*}(\eta)\right|=1$ as before. Then $\tau_{\Phi}\left(S_{m}\right)=\sigma_{n+m}\left(E_{\Phi}\right)$. Now, by applying (1) to the characteristic function of $S_{m}$, we obtain $\sigma_{n+m}\left(E_{\Phi}\right)=\mu(E)$ where $E=\left\{z \in B_{n}:|\varphi(z)|^{2}\left(1-|z|^{2}\right)=1\right\}$. Note that $E$ is the zero set of the nontrivial realanalytic function $|\varphi(z)|^{2}\left(1-|z|^{2}\right)-1$ on $B_{n}$ and thus $\mu(E)=0$. It is clear from (1) that $\tau_{\Phi}$ is rotation-invariant. In summary: $\tau_{\Phi}$ is a rotation-invariant measure on $B_{m}$. Thus, by Lemma 4.5, $\left\|\tau_{\Phi}\right\|_{C}=0$. In conclusion: $\Phi$ induces a compact composition operator $C_{\Phi}: H^{2}\left(B_{m}\right) \rightarrow H^{2}\left(B_{n+m}\right)$ by Corollary 5.2.

Remark 5.4. Let $\alpha>-1$ and define $d \mu_{\alpha}(z)=\left(1-|z|^{2}\right)^{\alpha} d V(z)$ (recall $V=V_{n}$ denotes the normalized volume measure on $B$ ). The $\alpha$-weighted Bergman space $A_{\alpha}^{2}(B)$ is the closed subspace of $L^{2}\left(\mu_{\alpha}\right)$ consisting of holomorphic functions. For a measure $\mu$ on $B$ 
define

and

$$
\|\mu\|_{\alpha}=\sup \frac{\mu(\Omega(\zeta, t))}{\mu_{\alpha}(\Omega(\zeta, t))} \quad(\zeta \in S, t>0)
$$

$$
\|\mu\|_{\alpha}=\lim \sup _{t>0} \sup _{\zeta \in s} \frac{\mu(\Omega(\zeta, t))}{\mu_{\alpha}(\Omega(\zeta, t))} .
$$

There is an analogue of the Carleson measure theorem in this context ([4], [6], [17]; also [9]): $\left\|J_{\mu}\right\|^{2} \cong\|\mu\|_{\alpha}$ where $J_{\mu}$ denotes the densely defined inclusion operator of $A_{\alpha}^{2}(B)$ into $L^{2}(\mu)$. Thus, if $C_{\Phi, \alpha}$ denotes the densely defined composition operator of $A_{\alpha}^{2}\left(B_{k}\right)$ into $A_{\alpha}^{2}\left(B_{n}\right)$ induced by a holomorphic map $\Phi: B_{n} \rightarrow B_{k}$ and if $\mu_{\Phi}=\mu_{\alpha}\left[\Phi^{-1}\right]$, then one easily obtains as before

$$
\left\|C_{\Phi, \alpha}\right\|^{2}=\left\|J_{\mu_{\Phi}}\right\|^{2} \cong\left\|\mu_{\Phi}\right\|_{\alpha} .
$$

Also, an easy modification of the proof of Proposition 3.4 shows $\left\|J_{\mu}\right\|\left\|^{2} \cong\right\| \mu \|_{\alpha}$ and therefore one has the following analogue of Theorem 5.1:

$$
\left\|C_{\Phi, \alpha}\right\|^{2}=\left\|J_{\mu_{\Phi}}\right\|^{2} \cong\left\|\mu_{\Phi}\right\|_{\alpha}
$$

for bounded operators $C_{\Phi, \alpha}$. In fact the above is much easier to prove because the boundary functions are not involved. Note that $C_{\Phi, \alpha}$ is a priori bounded for $k=1$. However, $C_{\Phi, \alpha}$ is not bounded for general $k$; such an example can be found in [9].

\section{REFERENCES}

1. L. Carleson, Interpolation by bounded analytic functions and the corona problem, Ann. of Math., 76 (1962), 547-559.

2. B. Choe, Composition property of holomorphic functions on the ball, Michigan Math. J., 36 (1989), 289-301. $225-240$.

3. B. Choe, Weights induced by homogeneous polynomials, Pacific J. Math., 139 (1989),

4. J. Cima and W. Wogen, A Carleson measure theorem for the Bergman space on the ball, J. Operator Theory, 7 (1982), 157-165.

5. C. Cowen, Linear fractional composition operators on $H^{2}$, Integral equations Operator theory, 11 (1988), 151-160.

6. W. Hasting, A Carleson measure theorem for Bergman spaces. Proc. Amer. Math. Soc., 52 (1975), 237-241. 65-78.

7. L. Hörmander, $L^{p}$ estimates for (pluri-)subharmonic functions, Math. Scand., 20 (1967), $237-248$.

8. B. MacCluer, Compact composition operators on $H^{p}\left(B_{n}\right)$, Michigan Math. J., 32 (1985),

9. B. MacCluer and J. Shapiro, Angular derivatives and compact composition operators on the Hardy and Bergman spaces, Canad. J. Math., 38 (1986), 878-906.

10. E. Nordgren, Composition operators, Canad. J. Math., 20 (1968), $442-449$. 13-17.

11. S. Power, Hörmander's Carleson theorem for the ball, Glasgow Math. J., 26 (1985),

12. W. Rudin, Composition with inner functions, Complex Variables, 4 (1984), 7-19.

13. W. Rudin, Function theory in the unit ball of $\mathbb{C}^{n}$, (Springer-Verlag, 1980).

14. W. Rudin, New constructions of functions holomorphic in the unit ball of $\mathbb{C}^{n}, \mathrm{CBMS}$ Regional Conf. Ser. in Math., No. 63, Amer. Math. Soc., 1986. 
15. J. Ryff, Subordinate $H^{p}$ functions, Duke Math. J., 33 (1966), 347-354.

16. J. Shapiro, The essential norm of a composition operator, Ann. of Math., 125 (1987), $375-404$.

17. D. Stegenga, Multiplers of the Dirichlet space, Illinois J. Math., 24 (1980), 113-139.

18. B. Tomaszewski, Interpolation and inner maps that preserve measure, J. Funct. Anal., 55 (1984), 63-67.

19. J. Weidman, Linear operators in Hilbert spaces, (Springer-Verlag, 1980).

Department of Mathematics

Korea Advanced Institute of Science and Technology, Kusong-Dong 373-1, Yusong-Gu,

TAEJON 305-701, KOREA 